\title{
Development and Validation of Nomogram to Predict Long-Term Prognosis of Critically III Patients with Acute Myocardial Infarction
}

\author{
Yiyang Tang (D' \\ Qin Chen' \\ Lihuang Zha' \\ Yilu Feng' \\ Xiaofang Zeng' \\ Zhenghui Liu ${ }^{2}$ \\ Famei $\mathrm{Li}^{\mathrm{l}}$ \\ Zaixin $\mathrm{Yu}^{\prime}$
}

'Department of Cardiology, Xiangya Hospital, Central South University, Changsha, Hunan, People's Republic of China; ${ }^{2}$ Department of Neurology, Xiangya Hospital, Central South University, Changsha, Hunan, People's Republic of China
Correspondence: Zaixin Yu Department of Cardiology, Xiangya Hospital, Central South University, Xiangya Road, Kaifu District, Changsha, 410008, People's Republic of China $\mathrm{Tel} / \mathrm{Fax}+86$ 73I-8432749

Email yuzaixin@।26.com
Purpose: Acute myocardial infarction (AMI) is a common cardiovascular disease with a poor prognosis. The aim of this study was to construct a nomogram for predicting the longterm survival of critically ill patients with AMI. This nomogram will help in assessing disease severity, guiding treatment, and improving prognosis.

Patients and Methods: The clinical data of patients with AMI were extracted from the MIMIC-III v1.4 database. Cox proportional hazards models were adopted to identify independent prognostic factors. A nomogram for predicting the long-term survival of these patients was developed on the basis of the results of multifactor analysis. The discriminative ability and accuracy of the multifactor analysis were evaluated according to concordance index (C-index) and calibration curves.

Results: A total of 1202 patients were included in the analysis. The patients were randomly divided into a training set $(n=841)$ and a validation set $(n=361)$. Multivariate analysis revealed that age, blood urea nitrogen, respiratory rate, hemoglobin, pneumonia, cardiogenic shock, dialysis, and mechanical ventilation, all of which were incorporated into the nomogram, were independent predictive factors of AMI. Moreover, the nomogram exhibited favorable performance in predicting the 4-year survival of patients with AMI. The training set and the validation set had a C-index of 0.789 (95\% confidence interval [CI]: 0.765-0.813) and 0.762 (95\% CI: 0.725-0.799), respectively.

Conclusion: The nomogram constructed herein can accurately predict the long-term survival of critically ill patients with AMI.

Keywords: acute myocardial infarction, nomogram, long-term prognosis, MIMIC-III, retrospective study

\section{Introduction}

Acute myocardial infarction (AMI) is defined as death or necrosis of myocardial cell caused by acute, severe, and sustained ischemia and hypoxia after coronary artery occlusion. It is the most serious subtype of coronary heart disease. ${ }^{1}$ According to NHANES data from 2013 to 2016, the overall prevalence of AMI among adults over 20 years old in the United States is 3.0\%, and an American will suffer from AMI approximately every $40 \mathrm{s.}^{2}$ Moreover, the prognosis of AMI is poor, with a 5 -year mortality rate as high as $51 \%$, thereby heavily placing a huge health and socioeconomic burden. ${ }^{3}$ Thus, risk factors of or models that can identify high-risk patients must be identified and developed to improve the prognosis of patients as timely and advanced interventions can administered. 
Several risk scores have been constructed to predict the prognosis of patients with AMI, among which the Thrombolysis in Myocardial Infarction ${ }^{4}$ and the Global Registry of Acute Coronary Events (GRACE) ${ }^{5}$ scores are the most widely used. However, these scores are only suitable for prognosis evaluation in the short term, such as in-hospital mortality, and long-term survival prediction on the basis of these risk scores for prognosis prediction in the short term is inaccurate. ${ }^{6}$ As enormous progress has been achieved in the diagnosis and treatment of AMI in the last few decades, especially the widespread adoption of emergency percutaneous coronary intervention, the survival time of patients with AMI has been substantially prolonged, and a prognostic model for predicting the longterm outcomes of patients must be developed. ${ }^{7}$

A nomogram, which is a tool for scoring risks in medical decision-making, is simple to operate and can be easily understood by visualizing the results of a prediction model. ${ }^{8}$ In clinical practice, the total score of patients can be calculated on the basis of the respective score of each predictor in the nomogram, and then the probability of specific disease-related outcomes can be obtained. Thus, nomograms have been successfully applied to various diseases, including septic acute kidney injury, ${ }^{9}$ acute type A aortic dissection, ${ }^{10}$ and heart failure. ${ }^{11}$ In the present study, the clinical records from a public database were used to conduct and validate the nomogram developed herein for predicting the long-term overall survival of critically ill patients with AMI.

\section{Materials and Methods}

\section{Data Source}

The clinical data for analysis was downloaded from the freely available critical care database, the Multiparameter Intelligent Monitoring in Intensive Care III version 1.4 (MIMIC-III v1.4), ${ }^{12}$ which is run by the Massachusetts Institute of Technology and funded by the National Institutes of Health (NIH). The database recorded detailed information from over forty thousand de-identified patients in Beth Israel Deaconess Medical Center between 2001 and 2012, including demographic data, vital signs, comorbidities, and laboratory tests, which provides reliable data resource for clinicians to conduct epidemiological studies. Besides, the database provides the patient's death time inside and outside the hospital from the hospital database or the social security database. The date of death outside the hospital is stored in two systems, namely the CareVue system with four years follow-up and the MetaVision with 90-days.

\section{Statement}

According to the requirements of the database, author Tang has completed the required training course, CITI "Data or Specimens Only Research" course, and passed the corresponding exams (record ID: 35980937) to get the access permission. Since this project has been approved by the institutional review boards of Massachusetts Institute of Technology (Cambridge, MA, USA) and Beth Israel Deaconess Medical Center (Boston, MA, USA) and the identifying elements related to patient privacy have been removed from the database, our research does not need to provide the additional approval of ethics committee.

\section{Participants and Design}

The process of participant inclusion and exclusion is presented in Figure 1. Among the 46,520 patients in the MIMIC-III database, only the patients who were first admitted to the intensive care unit (ICU) and diagnosed with AMI according to the 9th revision of the International Classification of Diseases Code (ICD-9) were included in this study. Patients $<18$ years old with ICU stay $<24 \mathrm{~h}$ and survival time $<0$ were excluded. Patients in the MetaVision system were also excluded because they had a short follow-up time. ${ }^{13}$ The prediction model was established by randomly selecting $70 \%$ of the eligible patients as the training set and the remaining $30 \%$ as the validation set, which was used to verify the prediction performance of the model. The primary clinical endpoint of this study was overall survival (OS), which was defined as the time from ICU admission to death or the last date of follow up (4 years).

\section{Data Extraction}

The clinical data of each patient, including demographic parameters, vital signs, comorbidities, laboratory test results, scoring systems, and interventions, were extracted from the MIMIC-III database by using the Structured Query Language in PostgreSQL tools (version 9.6). Demographic parameters mainly referred to age, gender, and ethnicity (Caucasian, Black, and others), whereas vital signs included body temperature, systolic blood pressure, diastolic blood pressure, heart rate (HR), respiratory rate (RR), and percutaneous oxygen saturation. The age of patients over 89 years old was fixed to 300 to protect their privacy, and the age of these patients was converted 


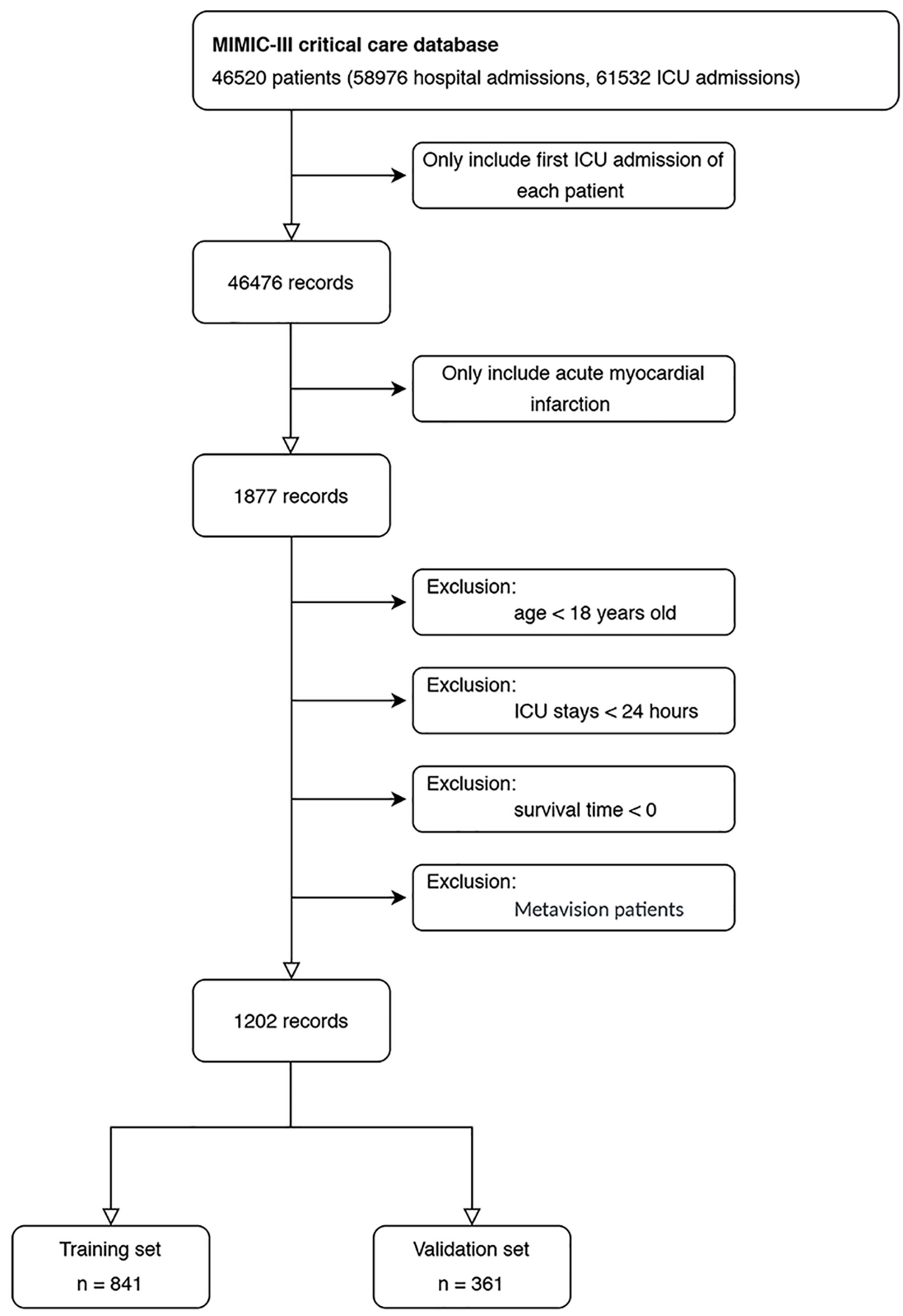

Figure I Workflow of the inclusion and exclusion of the study subjects.

Abbreviation: ICU, intensive care unit.

using the equation real age $=$ age $-300+89 .{ }^{14}$ Comorbidities were also extracted with their corresponding ICD-9 codes, including cardiogenic shock, cardiac arrest, congestive heart failure, valvular heart diseases, pulmonary circulation diseases, peripheral vascular diseases, hypertension, diabetes, pneumonia, respiratory failure, liver disease, renal failure, stroke, depression, and hypothyroidism. The Sequential Organ Failure Assessment score (SOFA) ${ }^{15}$ and the Simplified Acute Physiology Score II (SAPS II) ${ }^{16}$ were used to assess disease severity. These scores were calculated according to the physiological and laboratory parameters upon hospital admission. 
Laboratory tests included counting the number of or estimating the levels of white blood cells (WBC), hemoglobin, platelets, anion gap, sodium, potassium, chloride, bicarbonate, creatinine, blood urea nitrogen (BUN), prothrombin time, activated partial thromboplastin time, troponin $\mathrm{T}(\mathrm{cTnT})$, and lactate within the first $24 \mathrm{~h}$ after ICU admission. ${ }^{17,18}$ Interventions involved the use of vasopressor medicine, dialysis, mechanical ventilation, and percutaneous coronary intervention (PCI). Except for cTnT and lactate, the missing values of the other variables were all within $10 \%$, which were filled by multivariate multiple imputation with chained equations; by contrast, cTnT and lactate were regarded as dummy variables in the statistical analysis to reduce the possible bias of simple filling. ${ }^{19}$

\section{Statistical Analysis}

Continuous variables were presented in the form of mean \pm standard deviation (SD) or median (IQR) with KruskalWallis test for hypothesis testing. Categorical variables were expressed as numbers (percentages) and analyzed via Chi-square or Fisher's exact tests, as appropriate.

The clinical data of the patients in the training set were used to construct the nomogram. First, univariate Cox regression analysis was conducted to explore possible variables that may be related to the OS of patients with AMI. Multivariable Cox regression analysis with forward stepwise selection was then performed on the significant variables in the univariate analyses $(p<0.05)$. Finally, the nomogram was established to visualize the results of the multivariate analysis by using the rms package in R. Variance inflation factor (VIF) was calculated to test the collinearity between variables, with 2 as the threshold.

In the validation set, the risk scores of each patient based on the results of the training set were regarded as a variable to conduct Cox proportional hazard regression. Concordance index (C-index) was calculated with the Hmisc package in $\mathrm{R}$ to assess the discrimination of the model for prognosis. Calibration curves plotted via the bootstrap method with 1000 resampling were used to reflect the consistency between the actual probability and that predicted by the nomogram. The sensitivity and specificity of the nomogram, as well as the SOFA and SAPS II scores in predicting the long-term prognosis of critically ill patients with AMI, were evaluated by the area under the curve (AUC) value of the receiver operating characteristic (ROC) curve by using the timeROC package in R.

All statistical analyses were implemented through Stata 16.0 (StataCorp LLC, College Station, Tex) and
R software version 3.5 .3 (http://www.r-project.org/). $p<$ 0.05 (two-sided) was considered statistically significant.

\section{Results}

\section{Baseline Characteristics of Subjects}

After screening the study subjects according to the inclusion and exclusion criteria, a total of 1,202 patients with AMI were included in the study, of which 841 patients were randomly entered onto the training set, and 361 patients were analyzed as the validation set. Overall, most of patients were male $(770,64.1 \%)$ and Caucasian $(736,61.2 \%)$, and the age of the subjects was generally old, with a median of 68.9. The detailed clinical characteristics of the patients in the training and validation sets are listed in Table 1. No significant differences were observed in most variables between the two groups. The 4-year OS rate was $65.9 \%$, whereas that in the training and validation sets was $66.8 \%$ and $63.7 \%$, respectively.

\section{Univariate and Multivariate Analysis}

The Cox proportional hazard model was applied to identify the prognostic factors of patients with AMI. The variables in Table 1 were introduced into the univariate Cox regression analysis. A total of 30 variables, including age, gender, and hemoglobin, served as significant factors for the OS of patients with AMI. The results are summarized in Table 2. These variables with $p<0.05$ were further included in the multivariate Cox regression analysis. Via the stepwise forward regression method, age, RR, BUN, cardiogenic shock, dialysis, mechanical ventilation, hemoglobin, and pneumonia were finally identified as independent prognostic factors for the 4-year OS of patients with AMI (hazard ratio: $0.90-2.03, p<0.01$, Table 3 ). The VIF value of these variables was $>2$, indicating that they had no linear correlation.

\section{Construction and Validation of Nomogram}

On the basis of the results of multivariate analysis, the risk factors listed in Table 3 were used to construct a nomogram for 4-year OS (Figure 2). The prediction performance of the nomogram was further confirmed using the values of $\mathrm{C}$-index and calibration curves. The $\mathrm{C}$-index for $\mathrm{OS}$ prediction in the training and validation sets was 0.789 (95\% CI: $0.765-0.813)$ and $0.762(95 \%$ CI: 0.725-0.799), respectively. The results demonstrated 
Table I Characteristics of Critically III Patients with AMI in the Training and Validation Sets

\begin{tabular}{|c|c|c|c|}
\hline \multirow[b]{2}{*}{ Variable } & \multicolumn{2}{|c|}{ Cohort } & \multirow[b]{2}{*}{$p$ value } \\
\hline & $\begin{array}{l}\text { Training } \\
(n=84 I)\end{array}$ & $\begin{array}{l}\text { Validation } \\
(n=361)\end{array}$ & \\
\hline Age (years) & $68.2 \pm 13.7$ & $67.4 \pm 14.3$ & 0.477 \\
\hline \multicolumn{4}{|l|}{ Gender, n (\%) } \\
\hline Female & $303(36.0)$ & $127(35.7)$ & 0.922 \\
\hline Male & $538(64.0)$ & $232(64.3)$ & \\
\hline \multicolumn{4}{|l|}{ Ethnicity, n (\%) } \\
\hline Caucasian & $512(60.9)$ & $224(62.0)$ & 0.468 \\
\hline Black & $30(3.6)$ & $8(2.2)$ & \\
\hline Other & $299(35.6)$ & $129(35.7)$ & \\
\hline \multicolumn{4}{|l|}{ HR, n (\%), beats/minute } \\
\hline$\geq 70,<90$ & 414 (49.2\%) & I 82 (50.4\%) & 0.744 \\
\hline$<70$ & $182(21.6 \%)$ & 71 (19.7\%) & \\
\hline$\geq 90$ & 245 (29.1\%) & 108 (29.9\%) & \\
\hline \multicolumn{4}{|l|}{ RR, n (\%), beats/minute } \\
\hline$<20$ & 599 (7I.2\%) & 267 (74.0\%) & 0.332 \\
\hline$\geq 20$ & $242(28.8 \%)$ & $94(26.0 \%)$ & \\
\hline \multicolumn{4}{|l|}{ SBP, n (\%), $\mathrm{mmHg}$} \\
\hline$\geq 100,<120$ & $44 \mathrm{I}(52.4 \%)$ & 192 (53.2\%) & 0.968 \\
\hline$<100$ & 172 (20.5\%) & 72 (19.9\%) & \\
\hline$\geq 120$ & $228(27.1 \%)$ & 97 (26.9\%) & \\
\hline \multicolumn{4}{|l|}{ DBP, n (\%), mmHg } \\
\hline$\geq 55,<65$ & 352 (4I.9\%) & 158 (43.8\%) & 0.590 \\
\hline$<55$ & $265(31.5 \%)$ & 103 (28.5\%) & \\
\hline$\geq 65$ & $224(26.6 \%)$ & $100(27.7 \%)$ & \\
\hline $\mathrm{SpO}_{2}, \%$ & 97.8 (96.5-98.7) & $97.7(96.3-98.6)$ & 0.234 \\
\hline \multicolumn{4}{|l|}{ Temperature, $\mathrm{n}(\%),{ }^{\circ} \mathrm{C}$} \\
\hline$\geq 36,<37.5$ & 677 (80.5\%) & 296 (82.0\%) & 0.791 \\
\hline$<36$ & $49(5.8 \%)$ & $18(5.0 \%)$ & \\
\hline$\geq 37.5$ & $115(13.7 \%)$ & 47 (13.0\%) & \\
\hline Weight, $\mathrm{Kg}$ & $80.6 \pm 19.8$ & $80.5 \pm 19.2$ & 0.880 \\
\hline \multicolumn{4}{|l|}{ Scoring systems } \\
\hline SOFA & $3.0(1.0-6.0)$ & $3.0(1.0-5.0)$ & 0.431 \\
\hline SAPS II & $33.0(25.0-44.0)$ & $31.0(23.0-43.0)$ & 0.172 \\
\hline \multicolumn{4}{|l|}{ Comorbidities, n (\%) } \\
\hline Cardiogenic shock & $152(18.1)$ & $51(14.1)$ & 0.094 \\
\hline Cardiac arrest & $63(7.5)$ & $29(8.0)$ & 0.746 \\
\hline $\mathrm{CHF}$ & $70(8.3)$ & $25(6.9)$ & 0.410 \\
\hline Pulmonary circulation & $5(0.6)$ & $2(0.6)$ & 1.000 \\
\hline Peripheral vascular & $62(7.4)$ & $29(8.0)$ & 0.691 \\
\hline disease & & & \\
\hline Valvular heart disease & $25(3.0)$ & $7(1.9)$ & 0.308 \\
\hline Hypertension & $65(7.7)$ & $14(3.9)$ & 0.014 \\
\hline Diabetes & $223(26.5)$ & $68(18.8)$ & 0.004 \\
\hline Pneumonia & $116(13.8)$ & $40(11.1)$ & 0.200 \\
\hline Respiratory failure & $91(10.8)$ & $37(10.2)$ & 0.769 \\
\hline
\end{tabular}

(Continued)
Table I (Continued).

\begin{tabular}{|c|c|c|c|}
\hline \multirow[b]{2}{*}{ Variable } & \multicolumn{2}{|c|}{ Cohort } & \multirow[b]{2}{*}{$p$ value } \\
\hline & $\begin{array}{l}\text { Training } \\
(n=84 I)\end{array}$ & $\begin{array}{l}\text { Validation } \\
(n=361)\end{array}$ & \\
\hline Liver diseases & $12(1.4)$ & $3(0.8)$ & 0.394 \\
\hline Renal failure & $87(10.3)$ & $23(6.4)$ & 0.029 \\
\hline Stroke & $24(2.9)$ & $12(3.3)$ & 0.661 \\
\hline Depression & $16(1.9)$ & $9(2.5)$ & 0.511 \\
\hline Hypothyroidism & $19(2.3)$ & $25(6.9)$ & 0.285 \\
\hline \multicolumn{4}{|l|}{ Laboratory test } \\
\hline WBC (K/ul) & $11.8(9.5-15.0)$ & $12.0(9.5-14.7)$ & 0.876 \\
\hline Platelet (K/ul) & $\begin{array}{c}214.0(164.2- \\
268.0)\end{array}$ & $\begin{array}{c}218.0(168.0- \\
271.0)\end{array}$ & 0.288 \\
\hline Hemoglobin (g/dl) & $11.6(10.1-13.1)$ & $11.8(10.4-13.3)$ & 0.753 \\
\hline Creatinine (mg/dl) & $1.0(0.8-1.3)$ & $0.9(0.7-1.2)$ & 0.090 \\
\hline BUN (mg/dl) & $19.0(14.0-27.0)$ & $18.0(13.0-26.0)$ & 0.148 \\
\hline Sodium (mmol/L) & $\begin{array}{c}139.0(136.0- \\
140.0)\end{array}$ & $\begin{array}{c}138.0(136.0- \\
141.0)\end{array}$ & 0.711 \\
\hline Potassium (mmol/L) & $4.1(3.8-4.5)$ & $4.1(3.8-4.5)$ & 0.974 \\
\hline Chloride (mmol/L) & $\begin{array}{c}105.0(102.0- \\
108.0)\end{array}$ & $\begin{array}{c}105.0(102.0- \\
108.0)\end{array}$ & 0.307 \\
\hline Bicarbonate $(\mathrm{mmol} / \mathrm{L})$ & $23.0(20.0-25.0)$ & $23.0(21.0-25.0)$ & 0.437 \\
\hline PT (second) & $13.6(12.8-14.8)$ & $13.4(12.7-14.6)$ & 0.066 \\
\hline APTT (second) & $36.6(27.6-64.7)$ & $35.2(27.3-62.8)$ & 0.297 \\
\hline $\begin{array}{l}\text { cTnT, } \mathrm{n}(\%) \\
\quad<\mathrm{I} \mathrm{ng} / \mathrm{mL} \\
\geq \mathrm{I} \mathrm{ng} / \mathrm{mL} \\
\text { Site of infarction, } \mathrm{n}(\%)\end{array}$ & $\begin{array}{l}235(27.9) \\
187(22.2)\end{array}$ & $\begin{array}{l}\text { I0I (28.0) } \\
90(24.9)\end{array}$ & 0.554 \\
\hline Anterolateral wall & $59(7.0)$ & $24(6.6)$ & 0.471 \\
\hline Anterior wall & $249(29.6)$ & $117(32.4)$ & \\
\hline Inferolateral wall & $38(4.5)$ & $19(5.3)$ & \\
\hline Inferoposterior wall & $68(8.1)$ & $23(6.4)$ & \\
\hline Inferior wall & $233(27.7)$ & $110(30.5)$ & \\
\hline Lateral wall & $23(2.7)$ & $4(1.1)$ & \\
\hline Other specified sites & $27(3.2)$ & $9(2.5)$ & \\
\hline Unspecified sites & $144(17.1)$ & $55(15.2)$ & \\
\hline Dialysis, n (\%) & $45(5.4)$ & $10(2.8)$ & 0.050 \\
\hline Vasopressor, n (\%) & $139(16.5)$ & $65(18.0)$ & 0.532 \\
\hline Ventilation, n (\%) & $229(27.2)$ & $97(26.9)$ & 0.898 \\
\hline $\mathrm{PCl}, \mathrm{n}(\%)$ & $474(56.4)$ & $207(57.3)$ & 0.753 \\
\hline 4-year overall survival & $562(66.8)$ & $230(63.7)$ & 0.297 \\
\hline
\end{tabular}

Abbreviations: AMI, acute myocardial infarction; HR, heart rate; RR, respiratory rate; SBP, systolic blood pressure; DBP, diastolic blood pressure; $\mathrm{SpO}$, percutaneous oxygen saturation; SOFA, stroke, and malignancy. Calculate the sequential organ failure assessment score; SAPS II, simplified acute physiology score II; PCl, percutaneous coronary intervention. $\mathrm{CHF}$, congestive heart failure; WBC, white blood cell; BUN, blood urea nitrogen; PT, prothrombin time; APTT, activated partial thromboplastin time; $\mathrm{cTnT}$, troponin $\mathrm{T}$.

that the nomogram had a great discriminatory ability in predicting the long-term OS of patients with AMI. Moreover, the calibration curves indicated good consistency between the predicted and the observed 4-year OS rates (Figure 3). 
Table 2 Univariate Cox Regression Analysis of 4-Year Overall Survival in the Training Set

\begin{tabular}{|c|c|c|c|}
\hline \multirow[b]{2}{*}{ Variable } & \multicolumn{3}{|c|}{ Univariable Analysis } \\
\hline & HR & $95 \% \mathrm{Cl}$ & $P$ value \\
\hline Age (years) & 1.05 & $1.04-1.06$ & $<0.0001$ \\
\hline Gender, male & 0.65 & $0.5 \mathrm{I}-0.82$ & 0.0004 \\
\hline HR ( $\geq 90$ beats/minute) & 1.64 & $1.27-2.13$ & 0.0002 \\
\hline RR ( $\geq 20$ beats/minute) & 1.68 & $1.32-2.15$ & $<0.0001$ \\
\hline $\mathrm{SBP}(<100 \mathrm{mmHg})$ & 1.79 & $1.35-2.38$ & $<0.0001$ \\
\hline $\mathrm{DBP}(<55 \mathrm{mmHg})$ & 1.52 & $1.18-1.97$ & 0.0014 \\
\hline Temperature $\left(\geq 37.5^{\circ} \mathrm{C}\right)$ & 1.66 & $1.22,2.26$ & 0.0013 \\
\hline Weight & 0.98 & $0.98-0.99$ & $<0.0001$ \\
\hline Dialysis & 3.68 & $2.58-5.26$ & $<0.0001$ \\
\hline Vasopressor & 1.98 & $1.5 \mathrm{I}-2.60$ & $<0.0001$ \\
\hline Mechanical ventilation & 2.28 & $2.23-3.58$ & $<0.0001$ \\
\hline $\mathrm{PCl}$ & 0.60 & $0.48-0.76$ & $<0.0001$ \\
\hline Cardiogenic shock & 2.61 & $2.02-3.38$ & $<0.0001$ \\
\hline $\mathrm{CHF}$ & 3.10 & $2.27-4.22$ & $<0.0001$ \\
\hline Valvular heart disease & 1.89 & $1.10-3.23$ & 0.0205 \\
\hline Pulmonary circulation & 6.38 & $2.37-17.17$ & 0.0002 \\
\hline Hypertension & 1.82 & $1.27-2.60$ & 0.0011 \\
\hline Pneumonia & 2.88 & $2.20-3.77$ & $<0.0001$ \\
\hline Respiratory failure & 3.36 & $2.52-4.48$ & $<0.0001$ \\
\hline Renal failure & 2.18 & $1.60-2.97$ & $<0.0001$ \\
\hline Stroke & 2.48 & $1.45-4.24$ & 0.0010 \\
\hline Anterior wall infarction & 0.58 & $0.37-0.92$ & 0.0192 \\
\hline WBC & 1.04 & $1.02-1.06$ & 0.0005 \\
\hline Hemoglobin & 0.84 & $0.80-0.89$ & $<0.0001$ \\
\hline Potassium & 1.28 & $|.09-| .5 \mid$ & 0.0033 \\
\hline Bicarbonate & 0.91 & $0.89-0.94$ & $<0.0001$ \\
\hline BUN & 1.03 & $1.03-1.04$ & $<0.0001$ \\
\hline Creatinine & 1.31 & $1.24-1.39$ & $<0.0001$ \\
\hline PT & 1.05 & $1.02-1.07$ & 0.0011 \\
\hline Lactate $(\geq 2 \mathrm{mmol} / \mathrm{L})$ & 1.83 & $1.35-2.46$ & $<0.0001$ \\
\hline
\end{tabular}

Abbreviations: HR, heart rate; RR, respiratory rate; SBP, systolic blood pressure; DBP, diastolic blood pressure; $\mathrm{PCl}$, percutaneous coronary intervention; $\mathrm{CHF}$, congestive heart failure; WBC, white blood cell; BUN, blood urea nitrogen; PT, prothrombin time; $\mathrm{HR}$, hazard ratio; $\mathrm{Cl}$, confidence interval.

Table 3 Multivariate Cox Regression Analysis of 4-Year Overall Survival in the Training Set

\begin{tabular}{|l|c|c|c|}
\hline \multirow{2}{*}{ Variable } & \multicolumn{3}{|c|}{ Multivariate Analysis } \\
\cline { 2 - 4 } & HR & $\mathbf{9 5 \%} \mathbf{~ C I}$ & p value \\
\hline Age & 1.05 & $1.04-1.06$ & $<0.0001$ \\
Respiratory rate & 1.66 & $1.28-2.12$ & $<0.0001$ \\
Pneumonia & 1.62 & $1.21-2.17$ & 0.0010 \\
Blood urea nitrogen & 1.02 & $1.01-1.02$ & $<0.0001$ \\
Cardiogenic shock & 1.99 & $1.52-2.60$ & $<0.0001$ \\
Dialysis & 2.03 & $1.36-3.02$ & $<0.0001$ \\
Mechanical ventilation & 1.90 & $1.45-2.48$ & $<0.0001$ \\
Hemoglobin & 0.90 & $0.85-0.96$ & 0.0010 \\
\hline
\end{tabular}

Abbreviations: $\mathrm{HR}$, hazard ratio; $\mathrm{Cl}$, confidence interval.

\section{Comparison of the Predictive Accuracy of the Prediction Model}

By plotting the ROC curves and calculating the AUC, the performance of the nomogram, SOFA scores, and SAPS II scores in predicting the long-term prognosis of critically ill patients with AMI was compared. As shown in Figure 4, in the training set, the AUC of the nomogram, SOFA score, and SAPS II score was 0.841 (95\% CI: $0.813-$ $0.868), 0.710$ (95\% CI: $0.673-0.747)$, and $0.771(95 \%$ CI: $0.739-0.804)$, respectively. In the validation set, the AUC of the nomogram, SOFA score, and SAPS II score was 0.814 (95\% CI: 0.768-0.859), 0.726 (95\% CI: $0.673-$ $0.780)$, and 0.809 (95\% CI: 0.762-0.857), respectively. These results demonstrated that the nomogram constructed herein had the best predictive performance with the highest AUC of the ROC curve $(p<0.001)$. Thus, it can be used to predict the 4-year OS of patients with AMI.

\section{Discussion}

As the age of the global population becomes older, AMI has become one of the most common causes of deaths and disability. ${ }^{20}$ Therefore, precise risk stratification methods, individual treatment, and follow-up strategies are urgently needed for patients with AMI. A nomogram is a visual medical prediction model that can provide accurate and personalized predictions for patient OS, and it allows clinicians to make standardized clinical decisions. ${ }^{21}$

In the present study, on the basis of the large MIMICIII database, a nomogram was developed and validated to predict the 4-year OS of patients with AMI by using the variables screened by univariate and multivariate analyses, including age, RR, BUN, cardiogenic shock, dialysis, mechanical ventilation, hemoglobin, and pneumonia. Previous studies constructed nomograms for AMI, but these nomograms only focused on the short-term mortality of patients and the occurrence of complications, such as bleeding and acute kidney injury. ${ }^{22,23}$ To the best of our knowledge, this study was the first to establish a nomogram for predicting the long-term survival of patients with AMI. In our prediction model, the predictors integrated into the model were easy to obtain and calculate, including vital signs, such as RR, and routine laboratory tests, such as BUN. These predictors are readily available, especially in some economically underdeveloped areas.

Statistical analysis revealed that age, RR, BUN, cardiogenic shock, hemoglobin, pneumonia, and the use of 
Points

Age (years)

Hemoglobin (g/dl)
10
$+\quad-\quad 20$
40

50

60

70

80

90

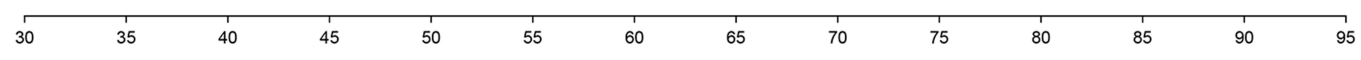

\begin{tabular}{lllllllll}
\hline 20 & 18 & 16 & 14 & 12 & 10 & 8 & 6 & 4
\end{tabular}

Blood urea nitrogen $(\mathrm{mg} / \mathrm{dl})$

Respiratory rate

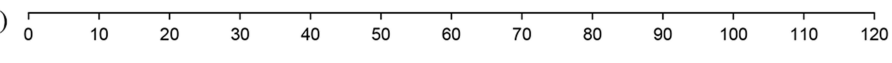

$\geq 20$

Pneumonia

$<20$

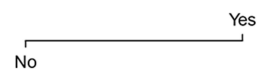

Dialysis

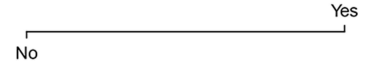

Mechanical ventilation

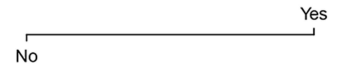

Cardiogenic shock

Yes

Total Points

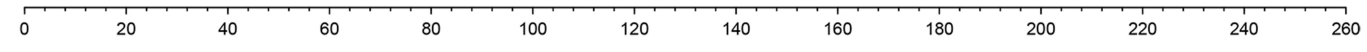

4-year OS rate

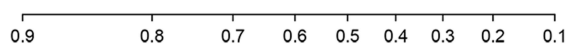

Figure 2 The nomogram for predicting the 4-year overall survival rate of patients with AMI. The nomogram included eight variables, including age, respiratory rate, blood urea nitrogen, cardiogenic shock, hemoglobin, pneumonia, and the use of dialysis and mechanical ventilation. In using the nomogram, a vertical line should be drawn upward from each variable to the "Points" line to obtain the score, and then the values are added to get the total score. Finally, a vertical line is drawn downward from the "Total Points" to obtain the 4-year overall survival of the patients with AMI.

Abbreviation: AMI, acute myocardial infarction.

dialysis and mechanical ventilation were independent factors to the long-term prognosis of AMI. This result was partly consistent with that of previous studies. Compared with young people, the elderly are often accompanied by structural and functional abnormalities of the heart and thus have a higher incidence of cardiovascular diseases. ${ }^{24}$ Age is also an independent predictor of the prognosis of patients with AMI. Chua et $\mathrm{al}^{25}$ demonstrated that short-term adverse events, including reinfarction, heart failure, and mortality, substantially increase with the increase in the age of patients undergoing PCI for ST-elevation myocardial infarction. Moreover, elderly patients with AMI have a higher middle- and long-term mortality than young people. ${ }^{26,27}$ As a very important vital sign, RR has been shown to be a risk factor of the poor prognosis of various cardiovascular diseases, including coronary artery disease. Barthel et $\mathrm{al}^{28}$ found that patients diagnosed with AMI with a high RR had a high risk of death, and an increase of four breaths per minute doubles the risk of death. The prognostic role of RR in AMI is independent of other existing risk models, such as GRACE score. Dommasch et $\mathrm{al}^{29}$ also clarified that RR can predict the possibility of gradual cardiac death in survivors of AMI. Both BUN and creatinine are the end-products of nitrogenous substances, and they are also the most commonly used indicators to reflect renal function in clinical practice. ${ }^{30}$ BUN levels are also affected by the status of low cardiac output, insufficient systemic and renal perfusion, and activation of the neurohumoral system, which usually occur in the early stages of AMI. After adjusting for creatinine and other potential covariables, elevated BUN levels upon admission are an important marker for inhospital and long-term mortality in patients with AMI. $^{31,32}$ Respiratory failure and acute kidney injury are the most common complications of AMI. Approximately $8 \%$ of patients with AMI require mechanical ventilation, and $3-4 \%$ of are treated with hemodialysis or other forms of renal replacement therapy. ${ }^{33,34}$ The use of these interventions has been proved to be strongly associated with the poor survival of patients, ${ }^{35}$ a finding similar to our results. 


\section{A}

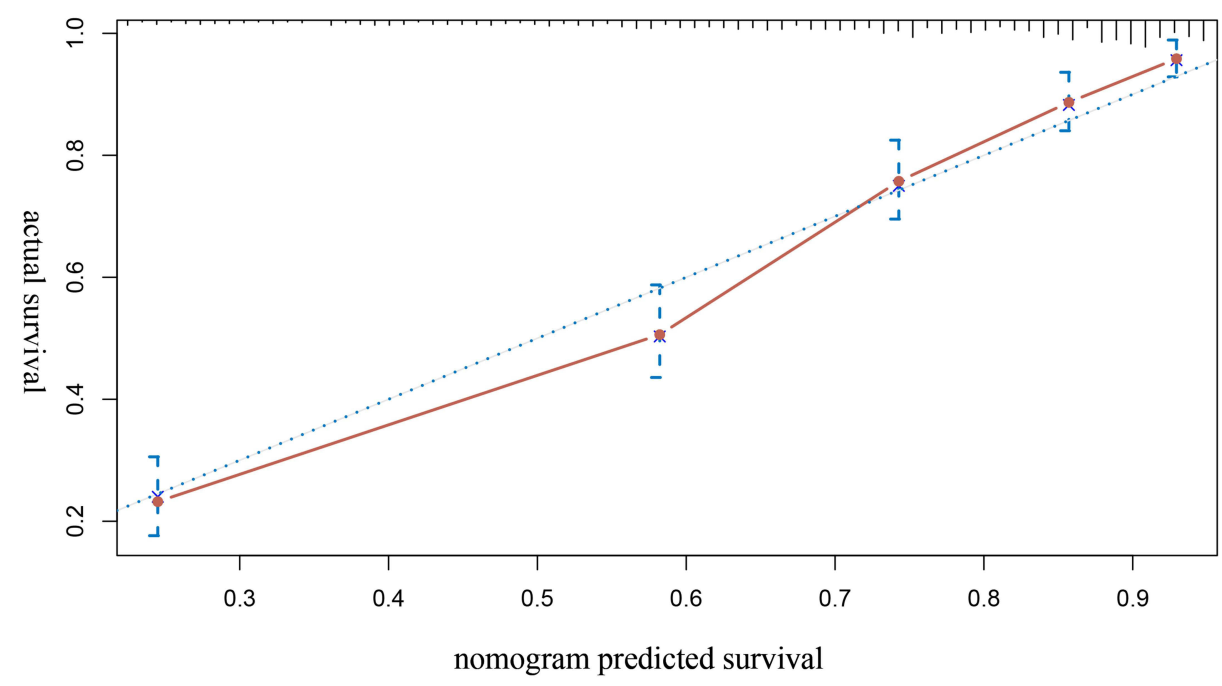

B

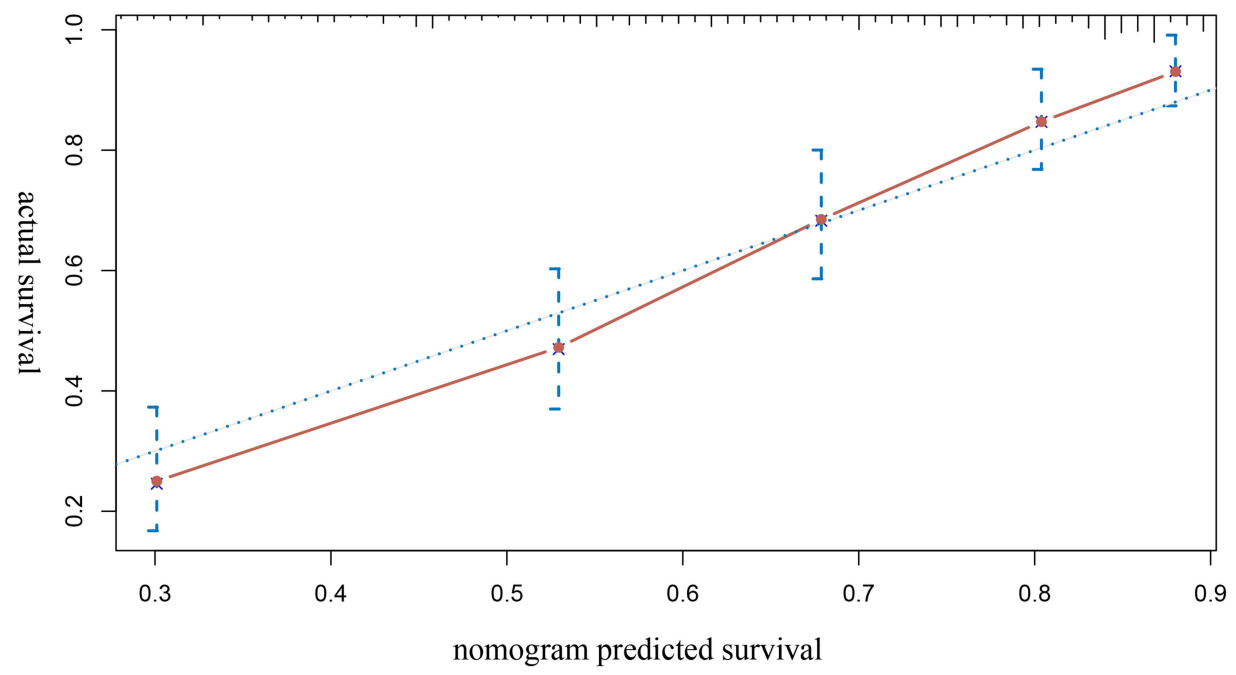

Figure 3 The calibration curve of the nomogram for predicting the 4-year overall survival in the training $(\mathbf{A})$ and the validation (B) sets. The dotted line represents the ideal curve where the predicted value is the same as the observed value. $\mathrm{X}$-axis: survival as predicted by the nomogram; $\mathrm{Y}$-axis: actual survival in the cohort.

In the research by Guo et $a 1,{ }^{36}$ a total of five risk factors for 30-day mortality of AMI, including age, HR, WBC, BUN level and bicarbonate level, were identified and used to establish a prognostic nomogram for shortterm prognosis. On the whole, the variables included in the nomogram for the short-term prognosis mainly reflect the acute disorder of internal environment under stress, such as the increase of HR, and the change of WBC, BUN and bicarbonate level, which are more likely to be closely related to the short-term prognosis of patients. In addition to these indicators reflecting acute stress such as RR, the nomogram model we constructed for long-term prognosis also included more indicators reflecting chronic failure of the body, such as the decrease of hemoglobin and the use of dialysis, which may be strongly related to the poor prognosis of patients, especially the long-term prognosis. According to the result of the research by Kunadian et al, ${ }^{37}$ anemia is strongly associated with poor short - and longterm outcomes of patients with AMI, especially in predicting long-term outcomes. Patients with anemia compared with those without anemia had significantly increased adverse event rates in hospital and at 1 month, especially at 1 year after discharge. Anemia was also an independent predictor of death at 1 year. Patients with chronic kidney 
A

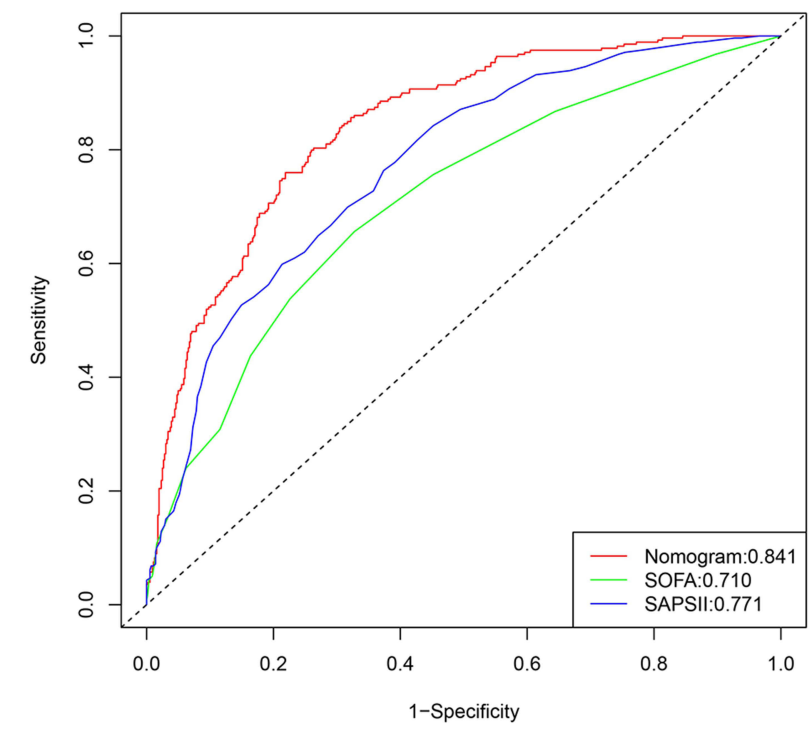

B

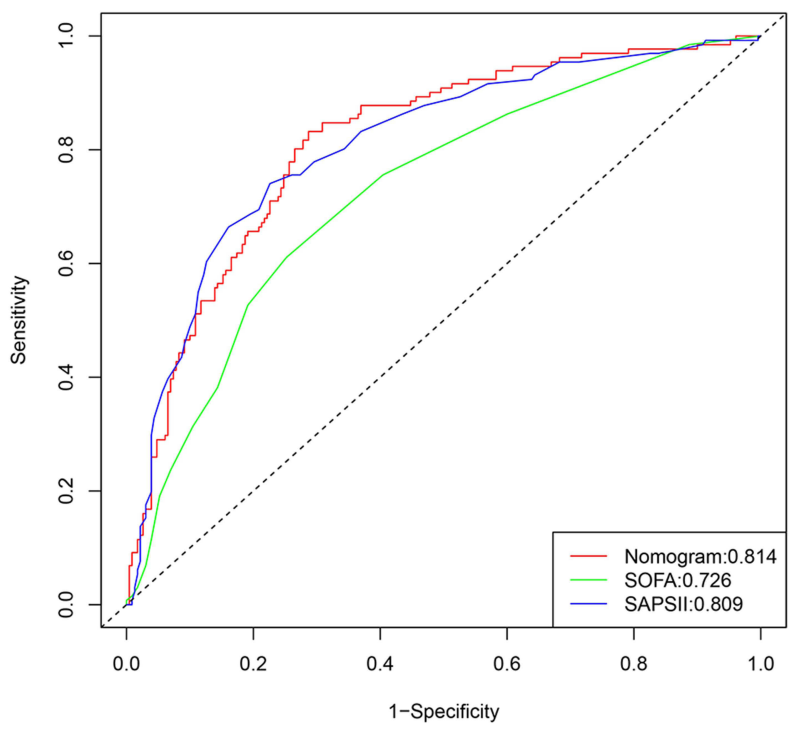

Figure 4 The ROC curve of the nomogram, SOFA and SAPS II scores for predicting the 4-year overall survival in the training (A) and validation (B) vs X-axis: I-specificity; Y-axis: sensitivity.

Abbreviations: ROC, receiver operating characteristic curve. AUC, area under the curve.

disease (CKD) are prone to the disorder of internal environment, which is closely related to the occurrence of myocardial infarction and its poor prognosis. The use of dialysis could efficiently remove toxins from the body and maintains a stable status for a period of time, but is still associated with poor long-term outcomes in patients with AMI. ${ }^{38} \mathrm{Fu}$ et al found that patients with non-dialysis CKD after AMI had longer stays in the ICU, longer hospital stays, and higher hospitalization costs compared to dialysis patients, while patients in the dialysis group had the highest 2-year mortality. ${ }^{39}$ In addition, the same variables included in the two nomograms are also different in the distribution of specific scores, which will lead to different prediction performance.

At the same time, the prediction performance of the proposed nomogram was also evaluated. The performance of a model largely depends on two aspects: it must have a good degree of discrimination, and it should have a certain degree of calibration. According to these standards, the nomogram constructed herein achieved a high C-index (0.762-0.789) and the AUC of the ROC curve (0.814-0.841). When the C-index or the AUC of the ROC curve of a prediction model is $>0.750$, the model has a good degree of discrimination, indicating that it can efficiently distinguish between high-risk and low-risk patients. Moreover, the calibration curve and the standard curve exhibited a high degree of coincidence, suggesting that the predicted results were highly consistent with the actual situation and that the predicted results were considerably reliable.

The SOFA and SAPS II scores are widely used in the clinical evaluation of disease severity in critically ill patients. ${ }^{16,40}$ Huang et $\mathrm{al}^{41}$ reported that the SOFA score can provide potentially valuable prognostic information on clinical outcome when applied to patients with AMI. The predictive power of the nomogram constructed herein, SOFA score, and SAPS II score in predicting the 4-year OS rate of critically ill patients with AMI was further illustrated by plotting its ROC curve. The AUC of the ROC curve was then calculated. Results showed that the nomogram constructed herein had a high predictive performance for long-term prognosis, with the highest AUC of the ROC curve. The bias caused by differences in treatment was avoided ${ }^{42,43}$ by conducting a sensitivity analysis focusing on the patients who received or did not undergo PCI. Regardless of whether the patients received PCI treatment or not, the nomogram constructed herein could effectively predict the long-term prognosis of these patients, with a high AUC of the ROC curve (Supplementary Figure 1). This result indicated that the nomogram is robust in predicting the long-term survival of patients. These results strongly indicated that the proposed nomogram can provide a reliable reference for clinical decision-making. 
This study has several limitations. First, the clinical data for analysis were extracted from a single-center institution, and the representativeness of the samples was limited to some extent. Second, vital signs, laboratory tests, and other variables were primarily derived from the data of patients within 24 $\mathrm{h}$ after ICU admission, and this step might have caused a certain degree of selection bias. Third, the indicators included in this study were mainly conventional and easily accessible parameters, and some specific indicators, such as $\mathrm{N}$-terminal pro brain natriuretic peptide and echocardiography parameters, were not included because of the large number of missing values, all of which might have reduced the accuracy of the model. Fourth, patients with type 1 and type 2 AMI were not distinguished owing to the limitations of the database. The development of a general model for simultaneously predicting the prognosis of patients with both types of AMI might have reduced the specificity of the nomogram because the two types have a different pathogenesis. Finally, the model was not externally validated using our own data.

\section{Conclusion}

The nomogram established herein can effectively predict the 4-year OS of critically ill patients with AMI. The validation results demonstrated that it has an accurate predictive performance and can provide a good reference for evaluating the long-term survival of these patients.

\section{Data Sharing Statement}

Publicly available datasets were analyzed in this study. This data can be extracted from Monitoring in Intensive Care Database III version 1.4 (MIMIC-III v.1.4) after passing on the required courses and obtaining the authorization.

\section{Acknowledgments}

Our study was supported by the National Natural Science Foundation of China $(81873416,82070055)$ and the Key Research and development program of Hunan Province (2020SK2065).

\section{Disclosure}

All authors declare that there is no conflict of interest.

\section{References}

1. Pei J, Wang X, Xing Z, et al. Association between admission systolic blood pressure and major adverse cardiovascular events in patients with acute myocardial infarction. PLoS One. 2020;15(6):e0234935. doi:10.1371/journal.pone. 0234935
2. Virani SS, Alonso A, Benjamin EJ, et al. Heart disease and stroke statistics-2020 update: a report from the American Heart Association. Circulation. 2020;141(9):e139-e596.

3. Kochar A, Chen AY, Sharma PP, et al. Long-term mortality of older patients with acute myocardial infarction treated in US clinical practice. J Am Heart Assoc. 2018;7(13):e007230. doi:10.1161/ JAHA.117.007230

4. Antman EM, Cohen M, Bernink PJ, et al. The TIMI risk score for unstable angina/non-ST elevation MI: a method for prognostication and therapeutic decision making. JAMA. 2000;284(7):835-842. doi:10.1001/jama.284.7.835

5. Granger CB, Goldberg RJ, Dabbous O, et al. Predictors of hospital mortality in the global registry of acute coronary events. Arch Intern Med. 2003;163(19):2345-2353. doi:10.1001/archinte.163.19.2345

6. Truong QA, Cannon CP, Zakai NA, et al. Thrombolysis in Myocardial Infarction (TIMI) Risk Index predicts long-term mortality and heart failure in patients with ST-elevation myocardial infarction in the TIMI 2 clinical trial. Am Heart J. 2009;157(4):673-679. doi:10.1016/j.ahj.2008.12.010

7. Ketchum ES, Dickstein K, Kjekshus J, et al. The Seattle Post Myocardial Infarction Model (SPIM): prediction of mortality after acute myocardial infarction with left ventricular dysfunction. Eur Heart J Acute Cardiovasc Care. 2014;3(1):46-55. doi:10.1177/ 2048872613502283

8. Balachandran VP, Gonen M, Smith JJ, DeMatteo RP. Nomograms in oncology: more than meets the eye. Lancet Oncol. 2015;16(4):e173e180. doi:10.1016/S1470-2045(14)71116-7

9. Deng F, Peng M, Li J, Chen Y, Zhang B, Zhao S. Nomogram to predict the risk of septic acute kidney injury in the first $24 \mathrm{~h}$ of admission: an analysis of intensive care unit data. Ren Fail. 2020;42 (1):428-436. doi:10.1080/0886022X.2020.1761832

10. Yang G, Zhou Y, He H, Pan X, Li X, Chai X. A nomogram for predicting in-hospital mortality in acute type A aortic dissection patients. $J$ Thorac Dis. 2020;12(3):264-275. doi:10.21037/ jtd.2020.01.41

11. Yang M, Tao L, An H, et al. A novel nomogram to predict all-cause readmission or death risk in Chinese elderly patients with heart failure. ESC Heart Fail. 2020;7(3):1015-1024. doi:10.1002/ehf2.12703

12. Johnson AE, Pollard TJ, Shen L, et al. MIMIC-III, a freely accessible critical care database. Sci Data. 2016;3:160035. doi:10.1038/ sdata.2016.35

13. Jia L, Cui S, Yang J, et al. Red blood cell distribution width predicts long-term mortality in critically ill patients with acute kidney injury: a retrospective database study. Sci Rep. 2020;10(1):4563. doi:10.1038/s41598-020-61516-y

14. Chen Q, Chen Q, Li L, et al. Serum anion gap on admission predicts intensive care unit mortality in patients with aortic aneurysm. Exp Ther Med. 2018;16(3):1766-1777.

15. Vincent JL, Moreno R, Takala J, et al. Working group on sepsis-related problems of the European Society of Intensive Care Medicine. The SOFA (Sepsis-related Organ Failure Assessment) score to describe organ dysfunction/failure. Intensive Care Med. 1996;22(7):707-710. doi:10.1007/BF01709751

16. Le Gall JR, Lemeshow S, Saulnier F. A new Simplified Acute Physiology Score (SAPS II) based on a European/North American multicenter study. JAMA. 1993;270(24):2957-2963. doi:10.1001/ jama.1993.03510240069035

17. Zhang W, Wang Y, Li W, Wang G. The association between the baseline and the change in neutrophil-to-lymphocyte ratio and short-term mortality in patients with acute respiratory distress syndrome. Front Med. 2021;8:636869. doi:10.3389/ fmed.2021.636869

18. Guo Q, Li H, Ouyang H, et al. Heart rate fluctuation and mortality in critically Ill myocardial infarction patients: a retrospective cohort study. Front Cardiovasc Med. 2021;8:577742. doi:10.3389/ fcvm.2021.577742 
19. Feng M, McSparron JI, Kien DT, et al. Transthoracic echocardiography and mortality in sepsis: analysis of the MIMIC-III database. Intensive Care Med. 2018;44(6):884-892. doi:10.1007/s00134-0185208-7

20. Mozaffarian D, Benjamin EJ, Go AS, et al. Heart disease and stroke statistics-2015 update: a report from the American Heart Association. Circulation. 2015;131(4):e29-e322.

21. Gao F, Zhang Q, Liu Y, et al. Nomogram prediction of individual prognosis of patients with acute-on-chronic hepatitis B liver failure. Dig Liver Dis. 2019;51(3):425-433. doi:10.1016/j.dld.2018.08.023

22. Zhou X, Sun Z, Zhuang Y, et al. Development and validation of nomogram to predict acute kidney injury in patients with acute myocardial infarction treated invasively. Sci Rep. 2018;8(1):9769. doi:10.1038/s41598-018-28088-4

23. Simonsson M, Winell H, Olsson $\mathrm{H}$, et al. Development and validation of a novel risk score for in-hospital major bleeding in acute myocardial infarction:-the SWEDEHEART score. J Am Heart Assoc. 2019;8 (5):e012157. doi:10.1161/JAHA.119.012157

24. Carro A, Kaski JC. Myocardial infarction in the elderly. Aging Dis. 2011;2(2):116-137.

25. Chua SK, Shyu KG, Hung HF, et al. Gender and age differences in short- and long-term outcomes following primary percutaneous coronary intervention for ST-elevation myocardial infarction. Acta Cardiol Sin. 2014;30(4):274-283.

26. Mehta RH, Rathore SS, Radford MJ, Wang Y, Wang Y, Krumholz HM. Acute myocardial infarction in the elderly: differences by age. J Am Coll Cardiol. 2001;38(3):736-741. doi:10.1016/ S0735-1097(01)01432-2

27. Plakht Y, Shiyovich A, Gilutz H. Predictors of long-term (10-year) mortality postmyocardial infarction: age-related differences. Soroka Acute Myocardial Infarction (SAMI) Project. J Cardiol. 2015;65 (3):216-223. doi:10.1016/j.jjcc.2014.06.001

28. Barthel P, Wensel R, Bauer A, et al. Respiratory rate predicts outcome after acute myocardial infarction: a prospective cohort study. Eur Heart J. 2013;34(22):1644-1650. doi:10.1093/eurheartj/ehs420

29. Dommasch M, Sinnecker D, Barthel P, et al. Nocturnal respiratory rate predicts non-sudden cardiac death in survivors of acute myocardial infarction. $J \mathrm{Am}$ Coll Cardiol. 2014;63(22):2432-2433. doi:10.1016/j.jacc.2014.02.525

30. Qian H, Tang C, Yan G. Predictive value of blood urea nitrogen/ creatinine ratio in the long-term prognosis of patients with acute myocardial infarction complicated with acute heart failure. Medicine. 2019;98(11):e14845. doi:10.1097/MD.0000000000014845

31. Horiuchi Y, Aoki J, Tanabe K, et al. A high level of blood urea nitrogen is a significant predictor for in-hospital mortality in patients with acute myocardial infarction. Int Heart J. 2018;59(2):263-271. doi:10.1536/ihj.17-009

32. Richter B, Sulzgruber P, Koller L, et al. Blood urea nitrogen has additive value beyond estimated glomerular filtration rate for prediction of long-term mortality in patients with acute myocardial infarction. Eur J Intern Med. 2019;59:84-90. doi:10.1016/j. ejim.2018.07.019
33. Lesage A, Ramakers M, Daubin C, et al. Complicated acute myocardial infarction requiring mechanical ventilation in the intensive care unit: prognostic factors of clinical outcome in a series of 157 patients. Crit Care Med. 2004;32(1):100-105. doi:10.1097/01. CCM.0000098605.58349.76

34. Marenzi G, Cosentino N, Marinetti A, et al. Renal replacement therapy in patients with acute myocardial infarction: rate of use, clinical predictors and relationship with in-hospital mortality. Int J Cardiol. 2017;230:255-261. doi:10.1016/j.ijcard.2016.12.130

35. Pesaro AE, Katz M, Katz JN, et al. Mechanical ventilation and clinical outcomes in patients with acute myocardial infarction: a retrospective observational study. PLoS One. 2016;11(3): e0151302. doi:10.1371/journal.pone.0151302

36. Guo Q, Wu M, Li H, et al. Development and validation of a prognostic nomogram for myocardial infarction patients in intensive care units: a retrospective cohort study. BMJ Open. 2020;10(12):e040291.

37. Kunadian V, Mehran R, Lincoff AM, et al. Effect of anemia on frequency of short- and long-term clinical events in acute coronary syndromes (from the Acute Catheterization and Urgent Intervention Triage Strategy Trial). Am J Cardiol. 2014;114(12):1823-1829. doi:10.1016/j.amjcard.2014.09.023

38. Herzog CA. Poor long-term survival of dialysis patients after acute myocardial infarction: bad treatment or bad disease? Am J Kidney Dis. 2000;35(6):1217-1220. doi:10.1016/S0272-6386(00)70061-8

39. Fu CM, Chang $\mathrm{CH}$, Lee $\mathrm{CC}$, et al. Impact of dialysis dependence on prognosis in patients with myocardial infarction: an 11-year population-based study. Medicine. 2018;97(6):e9833. doi:10.1097/ MD.0000000000009833

40. Allard J, Cotin S, Faure F, et al. SOFA-an open source framework for medical simulation. Stud Health Technol Inform. 2007;125:13-18.

41. Huang SS, Chen YH, Lu TM, Chen LC, Chen JW, Lin SJ. Application of the Sequential Organ Failure Assessment score for predicting mortality in patients with acute myocardial infarction. Resuscitation. 2012;83(5):591-595. doi:10.1016/j.resuscitation.2011.12.014

42. Blondal M, Ainla T, Marandi T, Baburin A, Rahu M, Eha J. Better outcomes for acute myocardial infarction patients first admitted to PCI hospitals in Estonia. Acta Cardiol. 2010;65(5):541-548. doi:10.1080/AC.65.5.2056241

43. Ribichini F, Steffenino G, Dellavalle A, et al. Comparison of thrombolytic therapy and primary coronary angioplasty with liberal stenting for inferior myocardial infarction with precordial ST-segment depression: immediate and long-term results of a randomized study. J Am Coll Cardiol. 1998;32(6):1687-1694. doi:10.1016/S07351097(98)00446-X
International Journal of General Medicine

\section{Publish your work in this journal}

The International Journal of General Medicine is an international, peer-reviewed open-access journal that focuses on general and internal medicine, pathogenesis, epidemiology, diagnosis, monitoring and treatment protocols. The journal is characterized by the rapid reporting of reviews, original research and clinical studies

\section{Dovepress}

across all disease areas. The manuscript management system is completely online and includes a very quick and fair peer-review system, which is all easy to use. Visit http://www.dovepress.com/ testimonials.php to read real quotes from published authors. 